\title{
Multiplicity distributions for jet parton showers in a medium
}

\author{
Nicolas Borghini ${ }^{\mathrm{a}}$ and Urs Achim Wiedemann ${ }^{\mathrm{a}, \mathrm{b}}$ \\ ${ }^{a}$ Physics Department, Theory Division, CERN, CH-1211 Geneva 23, Switzerland

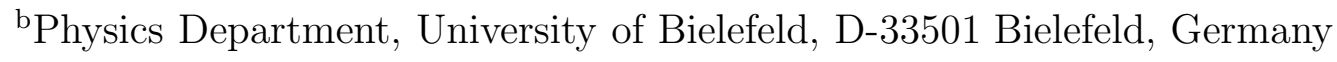

The "jet-quenching" interpretation of suppressed high- $p_{T}$ hadron production at RHIC implies that jet multiplicity distributions and jet-like particle correlations in heavy-ion collisions at RHIC and LHC differ strongly from those seen at $e^{+} e^{-}$or $p p(p \bar{p})$ colliders. Here, we present an approach for describing the changes induced by the medium, which implements jet quenching as a probabilistic medium-modified parton shower, treating leading and subleading parton splittings on an equal footing. We show that the strong suppression of single inclusive hadron spectra measured in $\mathrm{Au}-\mathrm{Au}$ collisions at RHIC implies a characteristic distortion of the single inclusive distribution of soft partons inside the jet. We determine, as a function of jet energy, to what extent the soft jet fragments can be measured above some momentum cut.

\section{INTRODUCTION}

One of the major discoveries of the first years of nucleus-nucleus collisions at RHIC is the observed deficit in the production of high transverse momentum particles if compared to $p p$ collisions [ [1]. The measurements strongly support the "jet quenching" interpretation, that high- $p_{T}$ partons traveling through the dense medium created in the collisions lose a significant fraction of their energy, through an enhanced radiation of gluons induced by the medium [ 2, 3, 4, before they hadronize outside the medium. Models based on this gluon radiation thus successfully account for the measured inclusive single-particle spectra and leading back-to-back two-particle correlations [ [5, 6].

Despite these successes of models based on medium-induced radiative energy loss which assume either multiple soft-momentum transfers [ 7, 8] or single hard scattering [ 3] from the medium - there is much room for technical improvements. In particular, standard formulations of parton energy loss consider only the medium-induced splitting of the leading parton, thus treating the splitting of leading and subleading partons differently. This makes it questionable to apply these approaches to the many measurements of jets and jet-like characteristics, such as particle-triggered multiplicity distributions, that are now gradually becoming available. Also, the current state of the art in applying mediummodified parton splittings involves numerically significant ad hoc corrections to maintain energy-momentum conservation.

In Ref. [ 9], we have proposed an alternative formulation of medium-induced parton energy loss. Our approach is the first that treats both leading and subleading parton split- 
tings on an equal footing. In addition, it conserves energy-momentum at each splitting. One of the main motivations for this approach is the possibility that these features may be more important than the precise treatment of coherence effects in medium-induced gluon emission, in particular for the discussion of high- $p_{T}$ particle correlations (for which an interesting, alternative extension of parton energy loss was proposed in Refs. [10, 11] ) and multiplicity distributions inside jets.

\section{FORMALISM}

Jets in $e^{+} e^{-}$or $p p / p \bar{p}$ collisions provide stringent tests of QCD. Color coherence, which results in destructive interference between partons with small momentum fractions $x=p / E_{\text {jet }}$ inside a shower, leads to unique predictions for the single inclusive distribution $D\left(x, Q^{2}\right)$ of partons inside a jet of energy and virtuality $E_{\text {jet }} \sim Q$ [ [12, 13]. The interference is actually equivalent, to double and single logarithmic accuracy in $\ln (1 / x)$ and $\ln \left(Q / \Lambda_{\text {eff }}\right)$ where $\Lambda_{\text {eff }}$ is an infrared cutoff, to an angular-ordered probabilistic parton shower with leading-order splitting functions. The resummed Modified Leading Logarithmic Approximation (MLLA) describes, to next-to-leading order $\sqrt{\alpha_{S}}$, the measured longitudinal distributions of hadrons $D^{h}\left(x, Q^{2}\right)$ in collisions over a wide energy range [ 14, 15, provided each parton is mapped locally onto a hadron ("Local Parton-Hadron Duality", LPHD) with a proportionality factor $\sim \mathcal{O}(1)$. As an example, Fig. 11 shows the distribution $D^{h}\left(x, Q^{2}\right)$ inside $17.5 \mathrm{GeV}$ jets, a typical jet energy at RHIC, measured in $e^{+} e^{-}$collisions by the TASSO Collaboration [14, together with the MLLA prediction.

We have developed a formalism for medium-distorted jets which reduces to the MLLA baseline in the absence of a medium [9]. With respect to the standard formalism of parton energy loss used currently in the analysis of RHIC data, the approach involves different approximations. Present model comparisons to RHIC data start with a medium-modified energy distribution of gluons $\mathrm{d} I^{\text {tot }}=\mathrm{d} I^{\text {vac }}+\mathrm{d} I^{\text {med }}$. Here, the vacuum contribution is double logarithmic $\mathrm{d} I^{\mathrm{vac}}=\frac{\alpha_{s}}{\pi^{2}} \frac{\mathrm{d} \omega}{\omega} \frac{\mathrm{d} \mathbf{k}}{\mathbf{k}^{2}}$, and its integral over $\mathbf{k}$ gives rise to the leading $\ln Q^{2}$ term in the DGLAP evolution equation. In contrast, the $\mathbf{k}$-integration of $\mathrm{d} I^{\text {med }}$ is infrared- and ultraviolet-safe [ [8, and leads to a geometrically enhanced $(\sim L)$ "higher twist" contribution $\propto \hat{q} L / Q^{2}$. However, these qualitatively different $Q^{2}$-dependences do not enter current comparisons to RHIC data. Instead, one considers the $\mathbf{k}$-integrated spectrum $\omega \frac{\mathrm{d} I^{\mathrm{med}}}{\mathrm{d} \omega}$ as an additional source of gluon radiation, whose dependence on $Q^{2}$ is neglected. Present approximations also do not include the further medium-induced splitting processes of subleading partons in the shower.

One improvement of this state of the art may be to replace the double differential $\mathrm{d} I^{\mathrm{vac}}$ by $\mathrm{d} I^{\text {tot }}$ in all leading and subleading splittings of a medium-modified parton shower. This requires a Monte-Carlo approach, which we intend to develop in future work. For the first analytical study, reported here, we have employed an additional approximation: We replaced the $\mathbf{k}$-integrated medium distribution $\omega \frac{\mathrm{d} I^{\text {med }}}{\mathrm{d} \omega}$ by a constant $f_{\text {med }}$. In the kinematic regime tested at RHIC, this turns out to be a fair approximation. We have then used

$\omega \frac{\mathrm{d} I^{\mathrm{med}}}{\mathrm{d} \omega}$ on the same level as $\omega \frac{\mathrm{d} I^{\mathrm{vac}}}{\mathrm{d} \omega}$, i.e., as a leading logarithmic correction [9]. With this ansatz, our formalism ensures energy-momentum conservation at each parton splitting, and treats all leading and subleading parton splittings on the same footing. The price we pay for an analytical treatment is the inaccurate handling of the $Q^{2}$-dependence (which is 

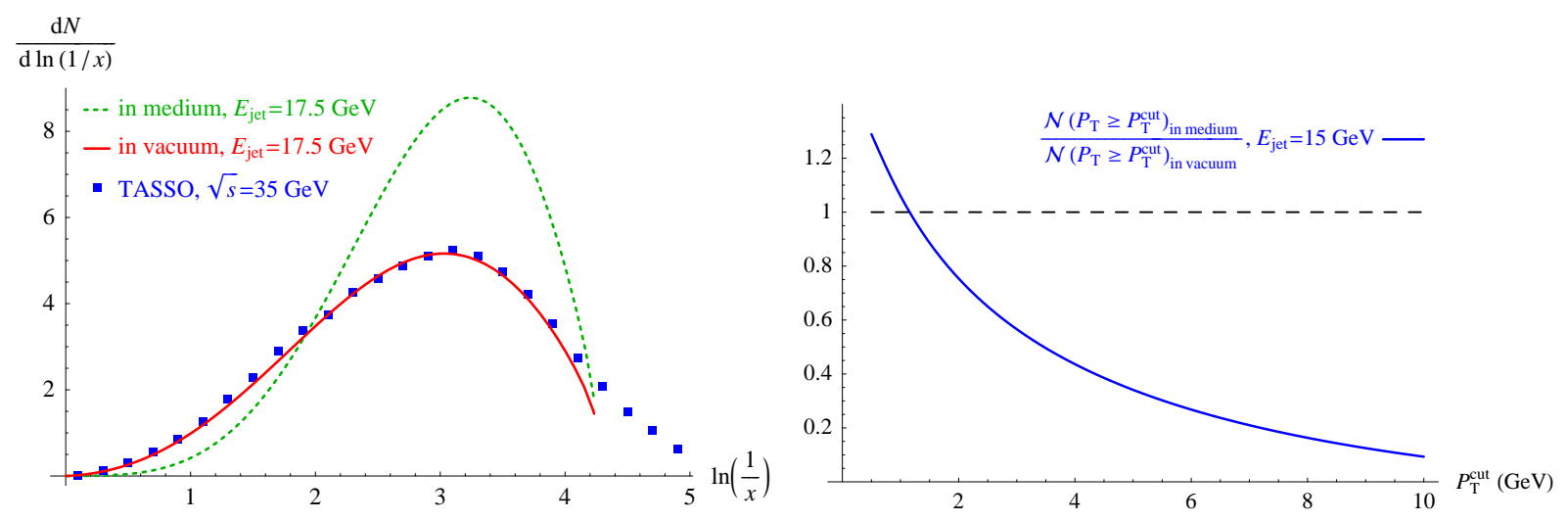

Figure 1. Single inclusive distribution of hadrons vs. $\ln \left(E_{\text {jet }} / p\right)$ for $E_{\text {jet }}=17.5 \mathrm{GeV}$ jets measured by TASSO in $e^{+} e^{-}$collisions Figure 2. Ratio of the multiplicities inside a jet in medium and in vacuum vs. the soft momentum cut above which the multiplicity and within MLLA (solid curve: $f_{\text {med }}=0$, is measured.

dashed curve: $\left.f_{\text {med }}=0.8\right)$.

not dealt with more properly in other formalisms) and the approximate treatment of the $\omega$-dependence. In a Monte-Carlo formulation of the same problem, we expect to remove the latter deficiencies. Moreover, the analytical results presented here will serve as a powerful consistency check for the correct implementation of splitting processes in the Monte-Carlo simulation.

\section{PHENOMENOLOGICAL PREDICTIONS}

In our analytical approach, we approximate the medium-induced contribution $\omega \frac{\mathrm{d} I_{\mathrm{med}}}{\mathrm{d} \omega}$ by a constant $f_{\text {med }}$ in the kinematically relevant range of $\omega$. Medium-modified parton splitting functions then differ from the standard ones by multiplying their singular contributions with $\left(1+f_{\text {med }}\right)$. This formulation allows us to follow the same line of technical arguments, used for the calculation of jet multiplicity distributions in the absence of a medium [13]. In Fig. 1, one sees the resulting in-vacuum and medium-distorted distributions $D^{h}\left(x, Q^{2}\right)$, plotted as a function of $\ln (1 / x)$, for jets with $E_{\text {jet }}=17.5 \mathrm{GeV}$. We chose the value $f_{\text {med }}=0.8$ to obtain in the same formalism a fair description of the suppression of high$p_{T}$ hadrons [9]. Figure 1]illustrates the effect of the medium, which results in a depletion of the number of particles at large $x$, and correspondingly a largely enhanced emission of particles at small $x$ : due to energy-momentum conservation in the parton shower, the energy which in the vacuum is taken by a single large- $x$ parton is redistributed over many small- $x$ partons in the presence of a medium.

Given the longitudinal multiplicity distribution inside a jet, a straightforward integration yields the number of particles inside the jet with momenta larger than a given cut, $\mathcal{N}\left(P_{T} \geq P_{T}^{\text {cut }}\right)$. We can then compute this multiplicity for jets with the same given energy both in the presence of medium effects (in which case, the cut gives some control on the high-multiplicity soft background of heavy-ion collisions at RHIC or LHC over which the jet develops) and in vacuum, and form their ratio. The latter, shown in Fig. 2 for jets with $E_{\text {jet }}=15 \mathrm{GeV}$ and medium effects modeled by $f_{\text {med }}=0.8$, is seen to be smaller than 
1 for $P_{T}^{\text {cut }}>1.5 \mathrm{GeV}$, while the medium-induced enhancement in soft particles becomes dominant for smaller values of the momentum cut. Although the medium-enhanced part of the multiplicity is a priori buried in the soft background of $\mathrm{Au}-\mathrm{Au}$ collisions at RHIC, this crossover value lies close to that reported by the STAR Collaboration in attempts at measuring the excess [16]. Note, however, that in our calculation the jet energy is known and fixed, while experimentally it spans a rather wide range.

\section{CONCLUSION}

We have reported a first step towards a description of parton showers in a medium, which conserves energy-momentum at each parton splitting, and deals in a symmetric way with all partons in the shower [9]. Our simplified analytical formalism is able to reproduce at least semi-quantitatively several characteristic features of RHIC data, such as the leading-hadron suppression and the enhanced soft-particle distribution associated to high- $p_{T}$ trigger particles. In the future, a Monte-Carlo implementation of this approach may be of great interest, since it would allow one to include more realistic mediuminduced splitting functions. Also, it would allow us to address the issue whether and how medium-induced parton energy loss depends on virtuality. This question is of particular importance for addressing the logarithmically wide kinematic $p_{T}$ range accessible at the LHC.

We thank Carlos Salgado for stimulating critical discussions.

\section{REFERENCES}

1. For a review, see P. Jacobs and X.-N. Wang, Prog. Part. Nucl. Phys. 54 (2005) 443, and references therein.

2. R. Baier, D. Schiff and B. G. Zakharov, Ann. Rev. Nucl. Part. Sci. 50 (2000) 37.

3. M. Gyulassy, I. Vitev, X.-N. Wang and B. W. Zhang, in Quark Gluon Plasma III, R. C. Hwa \& X.-N. Wang (eds.), World Scientific, Singapore, 2004, p. 123.

4. A. Kovner and U. A. Wiedemann, in Quark Gluon Plasma III, R. C. Hwa \& X.N. Wang (eds.), World Scientific, Singapore, 2004, p. 192.

5. A. Dainese, C. Loizides and G. Paic, Eur. Phys. J. C 38 (2005) 461.

6. K. J. Eskola, H. Honkanen, C. A. Salgado and U. A. Wiedemann, Nucl. Phys. A 747 (2005) 511.

7. R. Baier, Yu. L. Dokshitzer, A. H. Mueller, S. Peigné and D. Schiff, Nucl. Phys. B 484 (1997) 265.

8. C. A. Salgado and U. A. Wiedemann, Phys. Rev. D 68 (2003) 014008.

9. N. Borghini and U. A. Wiedemann, hep-ph/0506218.

10. A. Majumder and X.-N. Wang, Phys. Rev. D 70 (2004) 014007.

11. A. Majumder, E. Wang and X.-N. Wang, nucl-th/0412061.

12. A. H. Mueller, Nucl. Phys. B 213 (1983) 85.

13. Yu. L. Dokshitzer, V. A. Khoze and S. I. Troian, Adv. Ser. Direct. High Energy Phys. 5 (1988) 241.

14. W. Braunschweig et al. [TASSO Collaboration], Z. Phys. C 47 (1990) 187.

15. G. Abbiendi et al. [OPAL Collaboration], Eur. Phys. J. C 27 (2003) 467. 
16. J. Adams et al. [STAR Collaboration], nucl-ex/0501016. 